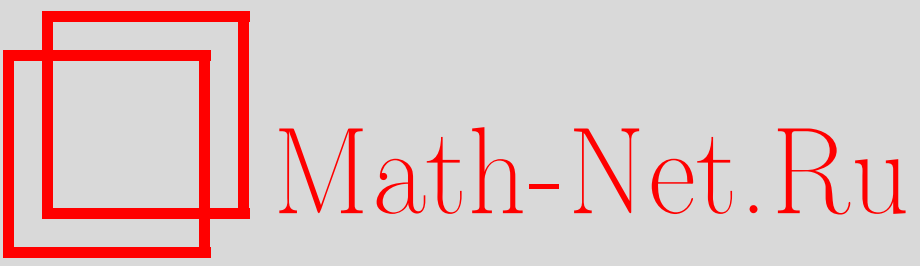

М. С. Маскина, Т. А. Жильников, Частные случаи квазипараллелограмма первого рода плоскости Лобачевского, Итоги науки и техн. Сер. Соврем. мат. и ее прил. Темат. обз., 2020, том 181, 66-73

DOI: https://doi.org/10.36535/0233-6723-2020-181-66-73

Использование Общероссийского математического портала Math-Net.Ru подразумевает, что вы прочитали и согласны с пользовательским соглашением

http://www.mathnet.ru/rus/agreement

Параметры загрузки:

IP: 18.208 .226 .222

26 апреля 2023 г., 17:08:12 


\title{
ЧАСТНЫЕ СЛУЧАИ КВАЗИПАРАЛЛЕЛОГРАММА ПЕРВОГО РОДА ПЛОСКОСТИ ЛОБАЧЕВСКОГО
}

\author{
(c) 2020 г. М. С. МАСКИНА, Т. А. ЖИЛЬНИКОВ
}

\begin{abstract}
Аннотация. Рассмотрены частные случаи квазипараллелограмма, которые получены при перенесении на плоскость Лобачевского характеристических свойств ромба, прямоугольника и квадрата евклидовой плоскости, связанных с их диагоналями. Доказано существование этих четырехугольников с применением модели Кели-Клейна в круге евклидовой плоскости.
\end{abstract}

Ключевые слова: плоскость Лобачевского, модель Кэли-Клейна, квазипараллелограмм, квазиромб.

\section{PARTICULAR CASES OF FIRST-KIND QUASI-PARALLELOGRAMS OF THE LOBACHEVSKY PLANE}

\author{
(c) 2020 M. S. MASKINA, T. A. ZHILNIKOV
}

\begin{abstract}
In this paper, we consider particular cases of quasi-parallelograms, which are obtained by transferring to the Lobachevsky plane various characteristic properties of rhombuses, rectangles, and squares of the Euclidean plane related with their diagonals. The existence of these quadrangles is proved by using the Cayley-Klein model in the circle of the Euclidean plane.
\end{abstract}

Keywords and phrases: Lobachevsky plane, Cayley-Klein model, quasi-parallelogram, quasirhombus.

AMS Subject Classification: 51F99

1. Введение. В классической литературе четырехугольники плоскости Лобачевского принято классифицировать по наличию и количеству у них прямых углов: четырехугольники Ламберт, Нестеровича, Саккери (см. [1-3]). Кроме дву- и трипрямоугольников часто упоминается гиперболический параллелограмма, понятие которого Н. И. Лобачевский ввел, перенеся его определение с евклидовой плоскости на гиперболическую (см. [4]).

В евклидовой геометрии параллелограмм и его частные случаи обладают целым рядом характеристических свойств, которые равносильны их определениям. Перенеся некоторые из этих свойств в качестве определений на плоскость Лобачевского, мы получили 4 новых типа четырехугольников, которые назвали квазипараллелограммами I-IV родов (см. [5]):

I: квазипараллелограммом I рода называется четырехугольник, у которого две противоположные стороны параллельны и равны;

II: квазипараллелограммом II рода называется четырехугольник, противоположные стороны которого попарно равны;

III: квазипараллелограммом III рода называется четырехугольник, у которого две противоположные стороны равны, а третья сторона образует с ними внутренние односторонние углы, сумма которых равна развернутому углу; 


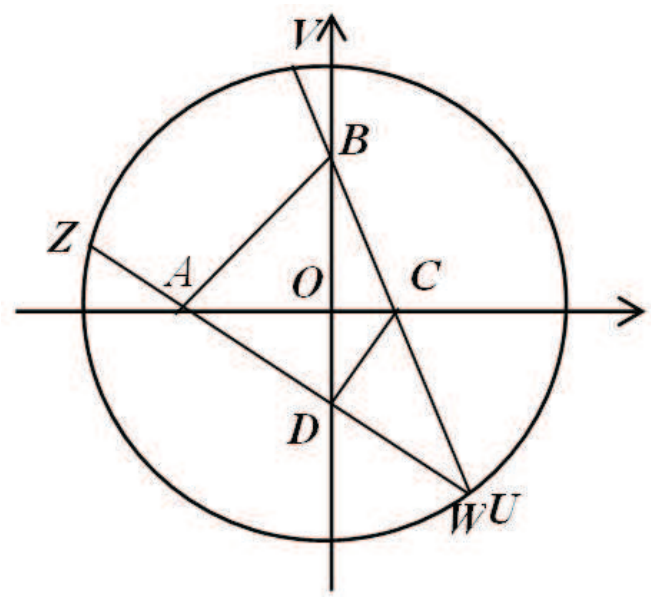

Рис. 1

IV: квазипараллелограммом IV рода называется четырехугольник, у которого каждая из двух пар углов, примыкающих к двум смежным сторонам, образует в сумме развернутый угол.

Авторами построены квазипараллелограммы каждого рода и доказано, что квазипараллелограмм II рода обладает следующими свойствами, каждое из которых является еще и его признаком не только на евклидовой, но и на гиперболической плоскости [6]:

(1) в квазипараллелограмме II рода противоположные углы которого попарно равны;

(2) в квазипараллелограмме II рода диагонали точкой пересечения делятся пополам;

(3) в квазипараллелограмме II рода две противоположные стороны равны, а прямая, проходящая через их середины, образует с ними внутренние односторонние углы, сумма которых равна развернутому углу;

(4) в квазипараллелограмме II рода одна из диагоналей образует равные внутренние накрест лежащие углы с обеими парами противоположных сторон;

(5) в квазипараллелограмме II рода две противоположные стороны равны, а одна из диагоналей при пересечении с ними образует равные внутренние накрест лежащие углы;

(6) в квазипараллелограмме II рода каждая из диагоналей при пересечении с одной из пар противоположных сторон образует равные внутренние накрест лежащие углы.

После получения 4 родов квазипараллелограммов нами поставлена задача изучения их частных случаев, а именно, четырехугольников, обладающих характеристическими свойствами ромба, прямоугольника или квадрата евклидовой плоскости. По аналогии будем называть эти четырехугольники квазиромбами, квазипрямоугольниками и квазиквадратами соответственно. Данная статья будет посвящена рассмотрению частных случаев квазипараллелограмма I рода.

2. Квазиромб I рода. В евклидовой плоскости ромб определяется как параллелограмм, у которого все стороны равны, а, следовательно, и противоположные стороны равны, но тогда мы получаем частный случай квазипараллелограмма II рода, поэтому в качестве определения квазиромба рассмотрим следующее.

Определение 1. Квазиромбом I рода называется квазипараллелограмм I рода, диагонали которого перпендикулярны.

Чтобы доказать, что такой четырехугольник существует, рассмотрим модель плоскости Лобачевского в круге евклидовой плоскости.

Пусть в евклидовой плоскости задана окружность $\omega$ уравнением $x^{2}+y^{2}=1$, а в ее внутренней области - квазипараллелограмм I рода $\boldsymbol{A B C D}$ так, что точка $\boldsymbol{O}$ пересечения его диагоналей совпадает с началом координат, точки $\boldsymbol{A}$ и $\boldsymbol{C}$ лежат на оси абсцисс, а $\boldsymbol{B}$ и $\boldsymbol{D}$ - на оси ординат. Тогда вершины квазиромба I рода $\boldsymbol{A} \boldsymbol{B} \boldsymbol{C D}$ будут иметь следующие координаты: $\boldsymbol{B}(0 ; b), \boldsymbol{A}(\bar{x} ; 0)$, 
$\boldsymbol{C}(a ; 0), \boldsymbol{D}(0 ; \bar{y})$, где числа $a, b, \bar{x}, \bar{y}$ по модулю меньше единицы (рис. 1$)$. Найдем условия, накладываемые на переменные $\bar{x}$ и $\bar{y}$, при выполнении которых длины отрезков $\boldsymbol{A D}$ и $\boldsymbol{B C}$ будут равны по Лобачевскому. Расстояние между точками $\boldsymbol{A}$ и $\boldsymbol{B}$ на плоскости Лобачевского определяется через метрику

$$
\delta(\boldsymbol{A} ; \boldsymbol{B})=\frac{1}{2} r|\ln (\boldsymbol{A B}, \boldsymbol{U} \boldsymbol{V})|,
$$

где $r$-радиус кривизны пространства, $\boldsymbol{U}$ и $\boldsymbol{V}$ - точки пересечения прямой $\boldsymbol{A B}$ с абсолютом $\boldsymbol{\omega}$, $(\boldsymbol{A} \boldsymbol{B}, \boldsymbol{U} \boldsymbol{V})$ - сложное отношение четырех точек одной прямой [1].

Наши исследования будем проводить по следующему плану:

1. Пусть $\boldsymbol{V}$ и $\boldsymbol{U}$ - точки пересечения прямой $\boldsymbol{B} \boldsymbol{C}$ с окружностью $\boldsymbol{\omega}$. Найдем координаты точек $\boldsymbol{V}$ и $\boldsymbol{U}$ и сложное отношение $(\boldsymbol{B} \boldsymbol{C}, \boldsymbol{V} \boldsymbol{U})$.

2. Пусть $\boldsymbol{W}$ и $\boldsymbol{Z}$ - точки пересечения прямой $\boldsymbol{A} \boldsymbol{D}$ с окружностью $\omega$. Найдем координаты точек $\boldsymbol{W}$ и $\boldsymbol{Z}$ и сложное отношение $(\boldsymbol{A} \boldsymbol{D}, \boldsymbol{W} \boldsymbol{Z})$.

3. Найдем условия равенства длин отрезков $\boldsymbol{B C}$ и $\boldsymbol{A D}$.

4. Найдем условия параллельности прямых $\boldsymbol{B C}$ и $\boldsymbol{A D}$.

1. Найдем координаты точек $\boldsymbol{V}$ и $\boldsymbol{U}$ и сложное отношение $(\boldsymbol{B C}, \boldsymbol{V} \boldsymbol{U})$.

Точки $\boldsymbol{V}$ и $\boldsymbol{U}$ принадлежат прямой $\boldsymbol{B} \boldsymbol{C}$, следовательно, существуют числа $\lambda_{1}$ и $\lambda_{2}$ такие, что точка $\boldsymbol{V}$ делит отрезок $\boldsymbol{B} \boldsymbol{C}$ в отношении $\lambda_{1}$, а $\boldsymbol{U}$-в отношении $\lambda_{2}$. Иначе говоря, выполняются равенства

$$
\overrightarrow{\boldsymbol{B} \boldsymbol{V}}=\lambda_{1} \overrightarrow{\boldsymbol{V} \boldsymbol{C}} \text { и } \overrightarrow{\boldsymbol{B U}}=\lambda_{2} \overrightarrow{\boldsymbol{U C}} .
$$

Тогда точки $\boldsymbol{V}$ и $\boldsymbol{U}$ имеют координаты:

$$
\boldsymbol{V}\left(\frac{\lambda_{1} a}{1+\lambda_{1}} ; \frac{b}{1+\lambda_{1}}\right), \quad \boldsymbol{U}\left(\frac{\lambda_{2} a}{1+\lambda_{2}} ; \frac{b}{1+\lambda_{2}}\right) .
$$

Но точки $\boldsymbol{V}$ и $\boldsymbol{U}$ принадлежат окружности $\omega$, следовательно, их координаты удовлетворяют ее уравнению, то есть числа $\lambda_{1}$ и $\lambda_{2}-$ корни уравнения:

$$
\left(\frac{\lambda a}{1+\lambda}\right)^{2}+\left(\frac{b}{1+\lambda}\right)^{2}=1
$$

или, иначе,

$$
(\lambda a)^{2}+b^{2}=(1+\lambda)^{2} .
$$

Приведем это квадратное уравнение к стандартному виду

$$
\lambda^{2}\left(a^{2}-1\right)-2 \lambda+b^{2}-1=0
$$

и решим его. Получим

$$
\lambda_{1}=\left(\frac{1-\sqrt{a^{2}+b^{2}-a^{2} b^{2}}}{a^{2}-1}\right), \quad \lambda_{2}=\left(\frac{1+\sqrt{a^{2}+b^{2}-a^{2} b^{2}}}{a^{2}-1}\right) .
$$

Тогда точки $\boldsymbol{V}$ и $\boldsymbol{U}$ имеют следующие координаты:

$$
\begin{aligned}
& \boldsymbol{V}\left(\frac{a-a \sqrt{a^{2}+b^{2}-a^{2} b^{2}}}{a^{2}-\sqrt{a^{2}+b^{2}-a^{2} b^{2}}} ; \frac{b\left(a^{2}-1\right)}{a^{2}-\sqrt{a^{2}+b^{2}-a^{2} b^{2}}}\right), \\
& \boldsymbol{U}\left(\frac{a+a \sqrt{a^{2}+b^{2}-a^{2} b^{2}}}{a^{2}+\sqrt{a^{2}+b^{2}-a^{2} b^{2}}} ; \frac{b\left(a^{2}-1\right)}{a^{2}+\sqrt{a^{2}+b^{2}-a^{2} b^{2}}}\right),
\end{aligned}
$$

а сложное отношение

$$
(\boldsymbol{B C}, \boldsymbol{V} \boldsymbol{U})=\frac{(\boldsymbol{B C}, \boldsymbol{V})}{(\boldsymbol{B C}, \boldsymbol{U})}=\frac{\lambda_{1}}{\lambda_{2}}=\frac{1-\sqrt{a^{2}+b^{2}-a^{2} b^{2}}}{1+\sqrt{a^{2}+b^{2}-a^{2} b^{2}}} .
$$


2. Найдем координаты точек $\boldsymbol{W}$ и $\boldsymbol{Z}$ и сложное отношение $(\boldsymbol{A} \boldsymbol{D}, \boldsymbol{W} \boldsymbol{Z})$. Повторив все рассуждения п. 1, получим, что точки $\boldsymbol{W}$ и $\boldsymbol{Z}$ имеют координаты:

а сложное отношение

$$
\begin{aligned}
& \boldsymbol{W}\left(\frac{\bar{x}-\bar{x} \sqrt{\bar{x}^{2}+\bar{y}^{2}-\bar{x}^{2} \bar{y}^{2}}}{\bar{x}^{2}-\sqrt{\bar{x}^{2}+\bar{y}^{2}-\bar{x}^{2} \bar{y}^{2}}} ; \frac{\bar{y}\left(\bar{x}^{2}-1\right)}{\bar{x}^{2}-\sqrt{\bar{x}^{2}+\bar{y}^{2}-\bar{x}^{2} \bar{y}^{2}}}\right), \\
& \boldsymbol{Z}\left(\frac{\bar{x}+\bar{x} \sqrt{\bar{x}^{2}+\bar{y}^{2}-\bar{x}^{2} \bar{y}^{2}}}{\bar{x}^{2}+\sqrt{\bar{x}^{2}+\bar{y}^{2}-\bar{x}^{2} \bar{y}^{2}}} ; \frac{\bar{y}\left(\bar{x}^{2}-1\right)}{\bar{x}^{2}+\sqrt{\bar{x}^{2}+\bar{y}^{2}-\bar{x}^{2} \bar{y}^{2}}}\right),
\end{aligned}
$$

$$
(\boldsymbol{A D}, \boldsymbol{W} \boldsymbol{Z})=\frac{(\boldsymbol{A D}, \boldsymbol{W})}{(\boldsymbol{A D}, \boldsymbol{Z})}=\frac{\lambda_{1}}{\lambda_{2}}=\frac{1-\sqrt{\bar{x}^{2}+\bar{y}^{2}-\bar{x}^{2} \bar{y}^{2}}}{1+\sqrt{\bar{x}^{2}+\bar{y}^{2}-\bar{x}^{2} \bar{y}^{2}}} .
$$

3. Так как длины отрезков $\boldsymbol{B C}$ и $\boldsymbol{A D}$ равны, то должно выполняться одно из равенств:

(a) $(\boldsymbol{A D}, \boldsymbol{W} \boldsymbol{Z})=(\boldsymbol{B C}, \boldsymbol{V} \boldsymbol{U})$;

(b) $(\boldsymbol{A D}, \boldsymbol{W} \boldsymbol{Z})=(\boldsymbol{C} \boldsymbol{B}, \boldsymbol{V} \boldsymbol{U})=(\boldsymbol{B C}, \boldsymbol{V} \boldsymbol{U})^{-1}$.

(а) Пусть $(\boldsymbol{A D}, \boldsymbol{W} \boldsymbol{Z})=(\boldsymbol{B C}, \boldsymbol{V} \boldsymbol{U})$. Тогда

$$
\frac{1-\sqrt{\bar{x}^{2}+\bar{y}^{2}-\bar{x}^{2} \bar{y}^{2}}}{1+\sqrt{\bar{x}^{2}+\bar{y}^{2}-\bar{x}^{2} \bar{y}^{2}}}=\frac{1-\sqrt{a^{2}+b^{2}-a^{2} b^{2}}}{1+\sqrt{a^{2}+b^{2}-a^{2} b^{2}}} .
$$

После ряда тождественных преобразований получим

$$
\sqrt{\bar{x}^{2}+\bar{y}^{2}-\bar{x}^{2} \bar{y}^{2}}=\sqrt{a^{2}+b^{2}-a^{2} b^{2}}
$$

откуда

(b) Пусть $(\boldsymbol{A D}, \boldsymbol{W} \boldsymbol{Z})=(\boldsymbol{C B}, \boldsymbol{V} \boldsymbol{U})$. Тогда

$$
\bar{y}= \pm \sqrt{\frac{a^{2}+b^{2}-a^{2} b^{2}-\bar{x}^{2}}{1-\bar{x}^{2}}}
$$

$$
\frac{1-\sqrt{\bar{x}^{2}+\bar{y}^{2}-\bar{x}^{2} \bar{y}^{2}}}{1+\sqrt{\bar{x}^{2}+\bar{y}^{2}-\bar{x}^{2} \bar{y}^{2}}}=\frac{1+\sqrt{a^{2}+b^{2}-a^{2} b^{2}}}{1-\sqrt{a^{2}+b^{2}-a^{2} b^{2}}},
$$

и после ряда тождественных преобразований получим уравнение

$$
\sqrt{\bar{x}^{2}+\bar{y}^{2}-\bar{x}^{2} \bar{y}^{2}}=-\sqrt{a^{2}+b^{2}-a^{2} b^{2}},
$$

что возможно только при условии

$$
\left\{\begin{aligned}
\bar{x}^{2}+\bar{y}^{2}-\bar{x}^{2} \bar{y}^{2}=0, \\
a^{2}+b^{2}-a^{2} b^{2}=0 .
\end{aligned}\right.
$$

Однако из второго уравнения данной системы следует, что

$$
b^{2}=\frac{a^{2}}{a^{2}-1},
$$

и, следовательно, $b^{2}<0$, (так как $|a|<1$ ), что невозможно.

4. Найдем условия параллельности прямых $\boldsymbol{B C}$ и $\boldsymbol{A D}$. Прямые $\boldsymbol{B C}$ и $\boldsymbol{A D}$ будут параллельны в направлении точки $\boldsymbol{U}$, если выполняется одно из следующих условий:

(a) точки $\boldsymbol{U}$ и $\boldsymbol{Z}$ совпадают;

(b) точки $\boldsymbol{U}$ и $\boldsymbol{W}$ совпадают.

(а) Рассмотрим первый случай: пусть точки $\boldsymbol{U}$ и $\boldsymbol{Z}$ совпадают. Тогда

$$
\frac{a+a \sqrt{a^{2}+b^{2}-a^{2} b^{2}}}{a^{2}+\sqrt{a^{2}+b^{2}-a^{2} b^{2}}}=\frac{\bar{x}+\bar{x} \sqrt{\bar{x}^{2}+\bar{y}^{2}-\bar{x}^{2} \bar{y}^{2}}}{\bar{x}^{2}+\sqrt{\bar{x}^{2}+\bar{y}^{2}-\bar{x}^{2} \bar{y}^{2}}} .
$$

Так как

$$
\sqrt{\bar{x}^{2}+\bar{y}^{2}-\bar{x}^{2} \bar{y}^{2}}=\sqrt{a^{2}+b^{2}-a^{2} b^{2}}
$$


то получим уравнение

$$
\frac{a\left(1+\sqrt{a^{2}+b^{2}-a^{2} b^{2}}\right)}{a^{2}+\sqrt{a^{2}+b^{2}-a^{2} b^{2}}}=\frac{\bar{x}\left(1+\sqrt{a^{2}+b^{2}-a^{2} b^{2}}\right)}{\bar{x}^{2}+\sqrt{a^{2}+b^{2}-a^{2} b^{2}}} .
$$

Отсюда после ряда тождественных преобразований получим уравнение

$$
\begin{aligned}
& a\left(1+\sqrt{a^{2}+b^{2}-a^{2} b^{2}}\right)\left(\bar{x}^{2}+\sqrt{a^{2}+b^{2}-a^{2} b^{2}}\right)- \\
& -\bar{x}\left(1+\sqrt{a^{2}+b^{2}-a^{2} b^{2}}\right)\left(a^{2}+\sqrt{a^{2}+b^{2}-a^{2} b^{2}}\right)=0 .
\end{aligned}
$$

и далее

$$
\left(1+\sqrt{a^{2}+b^{2}-a^{2} b^{2}}\right)(\bar{x}-a)\left(a \bar{x}-\sqrt{a^{2}+b^{2}-a^{2} b^{2}}\right)=0 .
$$

Корни последнего уравнения суть

$$
\bar{x}=a ; \quad \bar{x}=\frac{\sqrt{a^{2}+b^{2}-a^{2} b^{2}}}{a} .
$$

Оба этих корня не удовлетворяют условию задачи, так как первый приводит к вырождению

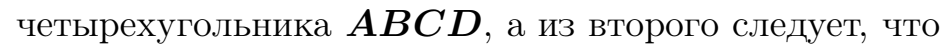

$$
a^{2} \bar{x}^{2}=a^{2}+b^{2}-a^{2} b^{2}
$$

откуда при учете $|\bar{x}|<1$ получаем, что

$$
b^{2}=\frac{a^{2}\left(\bar{x}^{2}-1\right)}{1-a^{2}}<0 .
$$

(b) Пусть точки $\boldsymbol{U}$ и $\boldsymbol{W}$ совпадают. Тогда

$$
\frac{a+a \sqrt{a^{2}+b^{2}-a^{2} b^{2}}}{a^{2}+\sqrt{a^{2}+b^{2}-a^{2} b^{2}}}=\frac{\bar{x}-\bar{x} \sqrt{\bar{x}^{2}+\bar{y}^{2}-\bar{x}^{2} \bar{y}^{2}}}{\bar{x}^{2}-\sqrt{\bar{x}^{2}+\bar{y}^{2}-\bar{x}^{2} \bar{y}^{2}}} .
$$

После замены

$$
\sqrt{\bar{x}^{2}+\bar{y}^{2}-\bar{x}^{2} \bar{y}^{2}}=\sqrt{a^{2}+b^{2}-a^{2} b^{2}}
$$

получим уравнение

$$
\frac{a\left(1+\sqrt{a^{2}+b^{2}-a^{2} b^{2}}\right)}{a^{2}+\sqrt{a^{2}+b^{2}-a^{2} b^{2}}}=\frac{\bar{x}\left(1-\sqrt{a^{2}+b^{2}-a^{2} b^{2}}\right)}{\bar{x}^{2}-\sqrt{a^{2}+b^{2}-a^{2} b^{2}}} .
$$

Отсюда после ряда тождественных преобразований получим уравнение

$$
\begin{aligned}
& a\left(1+\sqrt{a^{2}+b^{2}-a^{2} b^{2}}\right)\left(\bar{x}^{2}-\sqrt{a^{2}+b^{2}-a^{2} b^{2}}\right)- \\
& -\bar{x}\left(1-\sqrt{a^{2}+b^{2}-a^{2} b^{2}}\right)\left(a^{2}+\sqrt{a^{2}+b^{2}-a^{2} b^{2}}\right)=0 .
\end{aligned}
$$

Введя обозначение

$$
\Delta=a^{2}+b^{2}-a^{2} b^{2},
$$

получим

$$
\bar{x}^{2} a(1+\sqrt{\Delta})-\bar{x}(1-\sqrt{\Delta})\left(a^{2}+\sqrt{\Delta}\right)-a \sqrt{\Delta}(1+\sqrt{\Delta})=0 .
$$

Решим это уравнение:

$$
D=(1-\sqrt{\Delta})^{2}\left(a^{2}+\sqrt{\Delta}\right)^{2}+4 a^{2} \sqrt{\Delta}(1+\sqrt{\Delta})^{2}>0
$$


откуда

$$
\begin{aligned}
& \bar{x}_{1}=\frac{(1-\sqrt{\Delta})\left(a^{2}+\sqrt{\Delta}\right)-\sqrt{(1-\sqrt{\Delta})^{2}\left(a^{2}+\sqrt{\Delta}\right)^{2}+4 a^{2} \sqrt{\Delta}(1+\sqrt{\Delta})^{2}}}{2 a(1+\sqrt{\Delta})}, \\
& \bar{x}_{2}=\frac{(1-\sqrt{\Delta})\left(a^{2}+\sqrt{\Delta}\right)+\sqrt{(1-\sqrt{\Delta})^{2}\left(a^{2}+\sqrt{\Delta}\right)^{2}+4 a^{2} \sqrt{\Delta}(1+\sqrt{\Delta})^{2}}}{2 a(1+\sqrt{\Delta})} .
\end{aligned}
$$

Таким образом, мы доказали, что если четырехугольник $\boldsymbol{A} \boldsymbol{B} \boldsymbol{C D}$, вершины которого имеют координаты $\boldsymbol{A}(\bar{x} ; 0), \boldsymbol{B}(0 ; b), \boldsymbol{C}(a ; 0), \boldsymbol{D}(0 ; \bar{y})$, является квазиромбом I рода, то $\bar{y}$ вычисляется по формуле (1), а $\bar{x}$ - по одной из формул (2) или (3).

Докажем обратное утверждение: если вершины четырехугольника $\boldsymbol{A} \boldsymbol{B} \boldsymbol{C D}$ плоскости Лобачевского в данной интерпретации имеют координаты $\boldsymbol{A}(\bar{x} ; 0), \boldsymbol{B}(0 ; b), \boldsymbol{C}(a ; 0), \boldsymbol{D}(0 ; \bar{y})$, где числа $a, b$, $\bar{x}$ и $\bar{y}$ принадлежат интервалу $(-1 ; 1)$, то $\bar{x}$ вычисляется по формуле $(2)$ или (3), а $\bar{y}$ удовлетворяет выражению (1), то $\boldsymbol{A} \boldsymbol{B} \boldsymbol{C D}$ является квазиромбом I рода.

Действительно, так как $\bar{x}$ удовлетворяет выражению (2) или (3), то имеет место параллельность сторон $\boldsymbol{B C}$ и $\boldsymbol{A D}$ четырехугольника $\boldsymbol{A} \boldsymbol{B} \boldsymbol{C D}$. Так как ордината точки $\boldsymbol{D}$ находится по формуле (1), то выполняется равенство длин этих сторон. Мы доказали, что $\boldsymbol{A} \boldsymbol{B} \boldsymbol{C D}-$ квазипараллелограмм I рода. Координаты вершин этого четырехугольника говорят о перпендикулярности его диагоналей, а значит, $\boldsymbol{A} \boldsymbol{B} \boldsymbol{C D}$ является квазиромбом I рода.

Докажем существование квазиромба I рода. Пусть $a=1 / 2$, а $b=1 / \sqrt{2}$. Рассмотрим четырехугольник $\boldsymbol{A} \boldsymbol{B} \boldsymbol{C D}$, вершины которого имеют координаты $\boldsymbol{A}(\bar{x} ; 0), \boldsymbol{B}(0 ; b), \boldsymbol{C}(a ; 0), \boldsymbol{D}(0 ; \bar{y})$, где числа $\bar{x}$ и $\bar{y}$ принадлежат интервалу $(-1 ; 1)$.

Вычислим значения $\overline{x_{1}}$ и $\overline{x_{2}}$ по формулам $(2)$ и $(3)$.

$$
\begin{gathered}
\sqrt{\Delta}=\sqrt{a^{2}+b^{2}-a^{2} b^{2}}=\sqrt{\frac{1}{4}+\frac{1}{2}-\frac{1}{8}}=\frac{\sqrt{10}}{4} \\
\boldsymbol{D}=(1-\sqrt{\Delta})^{2}\left(a^{2}+\sqrt{\Delta}\right)^{2}+4 a^{2} \sqrt{\Delta}(1+\sqrt{\Delta})^{2}= \\
=\frac{(4-\sqrt{10})^{2}}{16} \frac{(1+\sqrt{10})^{2}}{16}+\frac{\sqrt{10}}{4} \frac{(4+\sqrt{10})^{2}}{16}=\frac{446+68 \sqrt{10}}{16^{2}} .
\end{gathered}
$$

Получим

$$
\begin{aligned}
& \bar{x}_{1}=\frac{(4-\sqrt{10})(1+\sqrt{10})-\sqrt{446+68 \sqrt{10}}}{16+4 \sqrt{10}} \approx-0,7757, \\
& \bar{x}_{2}=\frac{(4-\sqrt{10})(1+\sqrt{10})+\sqrt{446+68 \sqrt{10}}}{16+4 \sqrt{10}} \approx 1,0191>1 .
\end{aligned}
$$

По формуле (1)

$$
\bar{y}=-\sqrt{\frac{\Delta-\bar{x}^{2}}{1-\bar{x}^{2}}} \approx-0,6886 .
$$

Таким образом, все вершины четырехугольника $\boldsymbol{A} \boldsymbol{B} \boldsymbol{C}$ лежат во внутренней области окружности $\omega$, что доказывает существование квазиромба I рода.

3. Квазипрямоугольник I рода. В евклидовой плоскости прямоугольник определяется как параллелограмм, у которого все углы равны 90², а, следовательно, и противоположные углы равны, но тогда мы получаем частный случай квазипараллелограмма II рода, поэтому в качестве определения квазипрямоугольника рассмотрим следующее.

Определение 2. Квазипрямоугольником I рода называется квазипараллелограмм I рода, диагонали которого равны. 


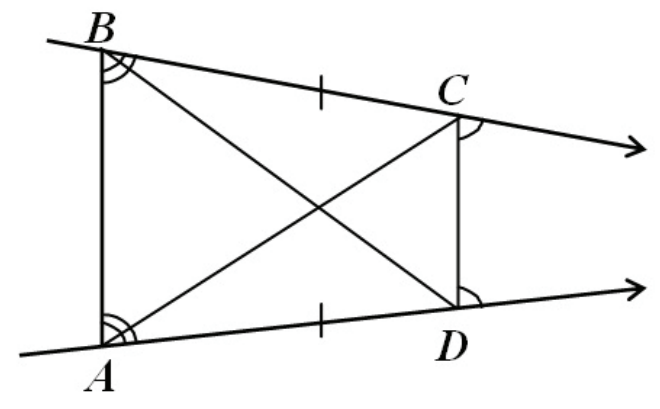

Рис. 2

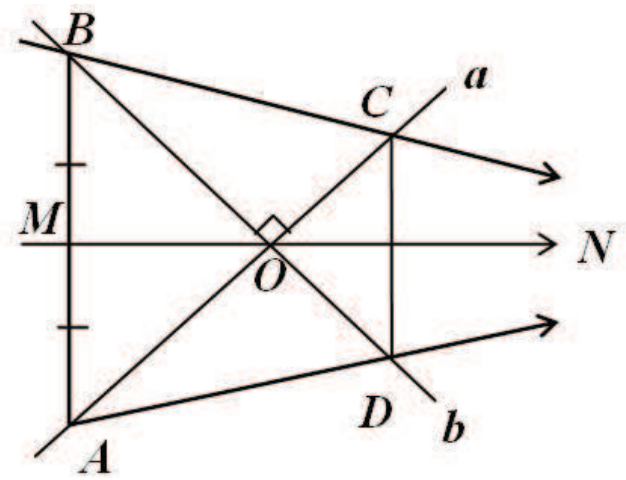

Рис. 3

Чтобы доказать существование такого четырехугольника, рассмотрим следующую теорему.

Теорема 1. Непараллельные между собой противоположные стороны квазипрямоугольника I рода является секущими равного наклона $к$ двум его параллельным сторонам.

Доказательство. Пусть в четырехугольнике $\boldsymbol{A B C D} \boldsymbol{A C}=\boldsymbol{B D}$ и $\boldsymbol{B C}=\boldsymbol{A D}$, тогда $\triangle \boldsymbol{A B C}=$ $\triangle \boldsymbol{B} \boldsymbol{A D}$ по трем сторонам(рис. 2), а тогда $\angle \boldsymbol{A B C}=\angle \boldsymbol{B} \boldsymbol{A D}$, то есть $\boldsymbol{A B}$ является секущей равного наклона к прямым $\boldsymbol{A C}$ и $\boldsymbol{B D}$. Аналогично из равенства $\triangle \boldsymbol{B C D}=\triangle \boldsymbol{A D C}$ следует, что $\angle \boldsymbol{B C D}=\angle \boldsymbol{A D C}$ и $\boldsymbol{C D}-$ секущая равного наклона к $\boldsymbol{A C}$ и $\boldsymbol{B D}$.

\section{Построение 1.}

1. Построим две параллельные прямые $a$ и $b$.

2. На прямой $b$ возьмем точки $\boldsymbol{B}$ и $\boldsymbol{C}$ (рис. 2).

3. Проведем через точки $\boldsymbol{B}$ и $\boldsymbol{C}$ секущие равного наклона $\boldsymbol{B} \boldsymbol{A}$ и $\boldsymbol{C} \boldsymbol{D}$ к прямым $a$ и $b$.

4. $\boldsymbol{A} \boldsymbol{B C D}$ - искомый четырехугольник.

Докажем, что полученный четырехугольник действительно будет квазипрямоугольником I poда. В самом деле, треугольники $\boldsymbol{A} \boldsymbol{B C}$ и $\boldsymbol{B} \boldsymbol{A D}$ равны по двум сторонам и углу (рис. 2), следовательно, $\boldsymbol{A C}=\boldsymbol{B} \boldsymbol{D}$.

4. Квазиквадрат I рода. В евклидовой плоскости квадрат определяется как четырехугольник, имеющий характеристические свойства и ромба, и прямоугольника. Оставим это утверждение верным и на плоскости Лобачевского.

Определение 3. Квазиквадратом I рода называется квазиромб I рода, диагонали которого равны.

Докажем построением, что такой четырехугольник существует.

\section{Построение 2.}

1. Построим две перпендикулярные прямые $a$ и $b$, пересекающиеся в точке $\boldsymbol{O}$.

2. На прямой $a$ возьмем произвольную точку $\boldsymbol{A}$ (рис. 3 ).

3. Возьмем на прямой $b$ точку $\boldsymbol{B}$ так, чтобы $\boldsymbol{A} \boldsymbol{O}=\boldsymbol{O B}$.

4. Проведем через середину $\boldsymbol{M}$ отрезка $\boldsymbol{A B}$ прямую $\boldsymbol{M O}$.

5. Проведем через точку $\boldsymbol{B}$ прямую $\boldsymbol{B} \boldsymbol{N}$, а через точку $\boldsymbol{A}$ прямую $\boldsymbol{A} \boldsymbol{N}$, параллельные прямой $\boldsymbol{M O}$ в данном направлении.

6. Обозначим точкой $\boldsymbol{C}$ точку пересечения прямых $a$ и $\boldsymbol{B} \boldsymbol{N}$, а точкой $\boldsymbol{D}$-точку пересечения прямых $b$ и $\boldsymbol{A} \boldsymbol{N}$.

7. Четырехугольник $\boldsymbol{A B C D}$ - искомый.

Заметим, что точки $\boldsymbol{C}$ и $\boldsymbol{D}$ действительно существуют. Так как точка $\boldsymbol{O}$ лежит внутри вырожденного треугольника $\boldsymbol{A} \boldsymbol{B N}$, то луч $\boldsymbol{A O}$ пересекает прямую $\boldsymbol{B N}$ [3], аналогично луч $\boldsymbol{B} \boldsymbol{O}$ пересекает прямую $\boldsymbol{A N}$. 
Докажем, что построенный четырехугольник является квазиквадратом I рода.

Доказательство. На рисунке 3 треугольник $\boldsymbol{A B O}$ - равнобедренный (по построению) и $\boldsymbol{M O}-$ его медиана, а значит и высота. Тогда $\angle \boldsymbol{M B N}=\boldsymbol{\Pi}(\boldsymbol{B} \boldsymbol{M})=\boldsymbol{\Pi}(\boldsymbol{A} \boldsymbol{M})=\angle \boldsymbol{M} \boldsymbol{A N}$ [3], то есть $\angle \boldsymbol{A} \boldsymbol{B C}=\angle \boldsymbol{B} \boldsymbol{A D}$, а тогда $\triangle \boldsymbol{A} \boldsymbol{B C}=\triangle \boldsymbol{B} \boldsymbol{A}$ (по стороне и двум углам). Следовательно, $\boldsymbol{A C}=$ $\boldsymbol{B D}$ и $\boldsymbol{A D}=\boldsymbol{B} \boldsymbol{C}$. Прямые $\boldsymbol{A D}$ и $\boldsymbol{B C}$ параллельны, а диагонали $\boldsymbol{A C}$ и $\boldsymbol{B D}$ перпендикулярны по построению, следовательно, построенный четырехугольник является квазиквадратом I рода.

Таким образом, через характеристические свойства ромба, прямоугольника и квадрата евклидовой плоскости, связанные с равенством или перпендикулярностью их диагоналей, введены понятия квазиромба, квазипрямоугольника и квазиквадрата І рода, являющихся частными случаями четырехугольника гиперболической плоскости, две стороны которого параллельны и равны, а также доказано существование этих четырехугольников.

\section{СПИСОК ЛИТЕРАТУРЫ}

1. Атанасян Л. С., Базылев В. Т. Геометрия. - М.: Просвещение, 1987.

2. Атанасян Л. С. Геометрия Лобачевского. - М.: Просвещение, 2001.

3. Каган В. Ф. Основания геометрии. - М.-Л.: ГИТТЛ, 1949.

4. Лобачевский Н. И. Полное собрание сочинений. Т. 3. - М.-Л.: ГИТЛЛ, 1951.

5. Маскина M. С. Обучение доказательству математически одаренных учащихся на факультативных курсах/ Дисс. на соиск. уч. степ. канд. пед. наук. - Саранск, 2003.

6. Маскина М. С., Купцов М. И. Частные случаи гиперболического параллелограмма плоскости Лобачевского// Тез. докл. Междунар. конф. «Геометрические методы в теории управления и математической физике». - Рязань: РГУ им. С. А. Есенина, 2018. - С. 53--54.

Маскина Мария Сергеевна

Академия права и управления ФСИН России, Рязань

E-mail: mariaya_maskina@mail.ru

Жильников Тимур Александрович

Академия права и управления ФСИН России, Рязань

E-mail: quadrus02@mail.ru 